\title{
Correction to: Halistanol sulfates I and J, new SIRT1-3 inhibitory steroid sulfates from a marine sponge of the genus Halichondria
}

\author{
Fumiaki Nakamura ${ }^{1}$ - Norio Kudo ${ }^{2}$ Yuki Tomachi ${ }^{1}$ Akiko Nakata ${ }^{2}$ Misao Takemoto ${ }^{2}$ Akihiro Ito $^{3,4}$. \\ Hodaka Tabei $^{1} \cdot$ Daisuke Arai $^{5} \cdot$ Nicole de Voogd ${ }^{6} \cdot$ Minoru Yoshida ${ }^{2,3,7} \cdot$ Yoichi Nakao $^{1,5} \cdot$ Nobuhiro Fusetani $^{5,8}$
}

Published online: 27 March 2018

(C) Japan Antibiotics Research Association 2018

Correction to: The Journal of Antibiotics (2018) 71, 273-278. https://doi.org/10.1038/ja.2017.145

The authors of the above article noticed the following errors in the publication of this paper in the Acknowledgement section.

In the Acknowledgements, the PDB id number" (PDB id 3GLU)" was wrong. It should have been read (PDB id 5Y4H).

Below is the corrected version of the subsection "Acknowledgements".

\section{Acknowledgements}

This paper is a part of the outcome of research performed under a Waseda University Grant-in-Aid for Scientific

F Nakamura and N Kudo contributed equally to this work.

Minoru Yoshida yoshidam@riken.jp

$\triangle$ Yoichi Nakao ayocha@waseda.jp

1 Department of Chemistry and Biochemistry, Graduate School of Advanced Science and Engineering, Waseda University, Shinjuku-ku, Tokyo, Japan

2 Seed Compounds Exploratory Unit for Drug Discovery Platform, RIKEN Center for Sustainable Resource Science, Wako, Saitama, Japan
Research, the Strategic Research Platforms for Private University, a Matching Fund Sub-sidy from the Ministry of Education, Science, Sports, and Technology (MEXT), Japan, JSPS KAKENHI Grant Number 26221204 and the Project for Development of Innovative Research on Cancer Therapeutics (P-Direct). This work was inspired by the JSPS Asian Chemical Biology Initiative. This work was performed under the approval of the Photon Factory Program Advisory Committee (Proposal Nos. 2013G674 and 2015G615). Atomic coordinates and structural factors have been deposited in the Protein Data Bank (PDB id 5Y4H).

3 Chemical Genomics Research Group, RIKEN Center for Sustainable Resource Science, Wako, Saitama, Japan

4 School of Life Sciences, Tokyo University of Pharmacy and Life Sciences, Hachioji, Tokyo, Japan

5 Research Institute for Science and Engineering, Waseda University, Shinjuku-ku, Tokyo, Japan

6 Naturalis Biodiversity Center, Leiden, The Netherlands

7 Department of Biotechnology, Graduate School of Agricultural Life Sciences, The University of Tokyo, Bunkyo-ku, Tokyo, Japan

8 Fisheries and Oceans Hakodate, Hakodate, Japan 